Orthopäde $2010 \cdot 39: 121$

DOI 10.1007/s00132-009-1516-5

Online publiziert: 30. Januar 2010

๑) Springer-Verlag 2010

\section{H. Zwipp}

Klinik und Poliklinik für Unfall- und Wiederherstellungschirurgie, Universitätsklinikum Carl Gustav Carus, Technische Universität Dresden

\section{Osteosyntheseverfahren}

\section{Historisches und Hochaktuelles}

Wenngleich steinzeitliche Funde unter Verkürzung verheilte Brüche langer Röhrenknochen nach vermutlich konservativer Behandlung belegen, kannten bereits die Azteken in Mexiko um 1400 n. Chr. offensichtlich die innere Schienung der Tibia mit einem sog. Feuerholz bei Versagen konservativer Maßnahmen. Die späteren Protagonisten der sog. Osteosynthese, d. h. des Zusammenführens gebrochener Knochenenden mit stabiler Implantatsicherung zur Bruchheilung, wie Malgaigne, Lister, von Langenbeck, König und Gluck sprachen von der sog. „Primärnaht“ des Knochens. Als Pioniere der Fixateur-externe-Osteosynthese gelten Wutzer (1847) und von Langenbeck (1855), für die intramedulläre Dieffenbach (1846), Riedinger (1881) und Bircher (1887). Als Vorreiter der Plattenosteosynthese gilt Hansmann (1886).

\section{( ) Lambotte verdanken wir den Begriff „Osteosynthese“}

Dem belgischen Chirurgen Albin Lambotte gebührt das Verdienst, die bis dahin geleisteten Erfahrungsberichte gesichtet und zielstrebig mit neuen Ideen verfolgt zu haben. Er fertigte in seiner eigenen Mechanikerwerkstatt selbst chirurgische Instrumente wie Knochenzangen, Drahtspanner, Raspatorien und Bohrer. Lambotte entwickelte nicht nur Osteosynthesetechniken mit Cerclagedrähten, sondern mit Fixateur externe, Zugschrauben und mit Platte. Letzere wurde von ihm „prothèse interne“ genannt. Die erste Anwendung eines Fixateur externe zur Osteosynthese am Femur erfolgte von ihm am 24.04.1902. Lambotte verdanken wir auch den Begriff „Osteosynthese“.
Während seit Küntscher (mit seiner ersten Oberschenkelmarknagelung am o9.11.1939) die Marknagelung (heute mit verfeinerter Technik) das Standardverfahren für diaphysäre Frakturen der langen Röhrenknochen ist, werden Schrauben und Platten vorzugsweise im gelenknahen Bereich verwandt.

Durch Einführung des neuen Facharztes für Orthopädie und Unfallchirurgie im Jahr 2003 entspricht es heute einem Selbstverständnis, dass unsere jungen Ärztinnen und Ärzte, die diese Facharztqualifikation anstreben, die Prinzipien der Frakturbehandlung mit primärer und sekundärer Knochenbruchheilung durch verschiedene Osteosyntheseverfahren erlernen.

In diesem Themenheft soll eine Synopsis zur Osteosynthese gegeben werden, die eingeleitet wird mit der Geschichte zur Entstehung diverser Osteosynthesen. Im zweiten Beitrag werden von Stephan Perren die Prinzipien der primären und sekundären Knochenheilung dargestellt. Die biomechanische Bedeutung der konventionellen Plattenosteosynthese bis hin zu den heute insbesondere am osteoporotischen Knochen favorisierten winkelstabilen Plattenosteosyntheseverfahren mit teils minimal-invasiver Technik wird in weiteren Beiträgen aufgezeigt. Auch heute noch kann bei Osteotomien, Arthrodesen, nichtheilenden Frakturen oder auch bei manchen gelenknahen Frakturen eine Klingenplattenosteosynthese ein sehr hilfreiches Verfahren sein. Die Kontroverse zur Stabilisierung langer Röhrenknochen durch aufgebohrte Marknagelung und durch die weniger traumatisierende unaufgebohrte Marknagelung wird in zwei weiteren Arbeiten ausführlich dar-

gestellt. Zuletzt wird deutlich, dass die Fixateur-externe-Osteosynthese auch heute noch ein approbates Osteosyntheseverfahren darstellt.

Möge die Übersicht der verschiedenen Osteosyntheseverfahren insbesondere der jungen Leserin und dem noch unerfahrenen Leser das notwendige Verständnis zu diesen technisch-anspruchsvollen, operativen Verfahren vermitteln.



H. Zwipp

\section{Korrespondenzadresse \\ H. Zwipp

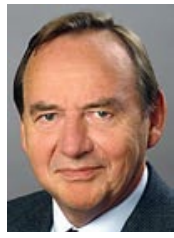 \\ Universitätsklinikum Carl Gustav Carus, an der Technischen Uni- versität Dresden, Klinik und Poli- klinik für Unfall- und Wieder- herstellungschirurgie Fetscherstr. 74, 01307 Dresden Hans.Zwipp@uniklinikum- dresden.de}

